\title{
Análise do Impacto de Chuvas na Velocidade Média do Transporte Público Coletivo de Ônibus em Recife
}

\author{
Alexandre S. G. Vianna, Michael O. Cruz, Luciano Barbosa, Kiev Gama
}

${ }^{1}$ Centro de Informática - Universidade Federal de Pernambuco (UFPE) - Recife - PE

Av. Jornalista Aníbal Fernandes S/N - 50740-560 - Recife - PE - Brasil

\{asgv, moc, luciano,kiev\}@cin.ufpe.br

\begin{abstract}
The adverse weather conditions represent an emblematic element that adversely affects the quality of public transport, particularly in tropical climate regions the rains constitute the main climatic event of this type. This paper explores the relationship between the rainfall episodes and the changes in the behavior of the average speed of public transport buses in the city of Recife. The work encompasses the use of descriptive statistics techniques to evaluate the itinerary of buses, positioning and speed data in contrast with the rainfall data recorded in the rain gauges scattered throughout the city of Recife. It is detailed in this study the analysis of regions known for traffic problems on rainy day. The results are presented and discussed in the paper.
\end{abstract}

Resumo. As condições climáticas adversas representam um fator emblemático que afeta negativamente a qualidade do transporte público, especialmente em regiões de clima tropical as chuvas são o principal evento climático deste tipo. Este artigo explora as relações entre eventos de chuva e o comportamento da velocidade média dos ônibus de transporte público na cidade do Recife. O trabalho envolve o uso de técnicas de estatística descritiva para analisar dados do itinerário, posicionamento e velocidade dos ônibus em contraste com os dados de precipitação em estações pluviométricas espalhadas pela cidade do Recife. Foram detalhadas as análises de locais conhecidos por problemas de trânsito em dias de chuva, os resultados são apresentados e discutidos no artigo.

\section{Introdução}

A influência do clima na dinâmica das redes viárias pode ter diferentes tipos de impacto nas cidades. Alguns estudos mostram correlações entre mudanças climáticas e o número de acidentes [Keay and Simmonds 2006], [Songchitruksa and Balke 2006], [Koetse and Rietveld 2009], enquanto outros mostram que o nível de congestionamentos pode ser agravado [Hofmann and O'Mahony 2005], [Smith et al. 2004]. Os impactos do clima afetam fatores econômicos, comportamento do tráfego, mudanças de itinerário, rotas, entre outros.

Avaliar esses impactos e obter informações que deem suporte para melhorar as tomadas de decisões na gestão do trânsito é um fator importante. Por exemplo, o governo britânico, por meio do Departamento de Transporte ${ }^{1}$, tem analisado vários dados de trânsito, desde 1950, como acidentes, congestionamentos, nível de poluição de forma a melhorar tomadas de decisões relacionadas ao tráfego.

Nos últimos anos, dados de GPS (Global Position System) têm sido coletados com maior facilidade graças à popularização de aparelhos celulares e dispositivos de IoTs

\footnotetext{
${ }^{1}$ https://www.gov.uk/government/uploads/system/uploads/attachment_data/file/661933/tsgb-2017report-summaries.pdf
} 
(Internet of Things). Muitas iniciativas existem para tornarem alguns desses dados abertos como Bikely ${ }^{2}$, Microsoft Geolife ${ }^{3}$ e Facebook ${ }^{4}$.

A massiva quantidade de dados gerados por dispositivos com GPS dá a oportunidade de analisar e entender a dinâmica do tráfego viário, além de favorecer o desenvolvimento de vários tipos de aplicações na área de ITS (Intelligent Transport System) como mineração de rotas [Savage et al. 2010], descobertas de sub-trajetórias [Pelekis et al. 2007], detecção de anomalias [Pan et al. 2013] etc. Além dos dados de trajetórias, outros dados de diferentes naturezas podem ser analisados conjuntamente como dados de clima, índices de poluentes, acidentes, ou seja, o volume de dados e a sua pluralidade caracteriza o que se chama de Big Data e, diante disso, dá a oportunidade de análise e criação de aplicações sobre dados que outrora não seria possível.

Neste trabalho, pretende-se fazer uma análise inicial do impacto das chuvas no serviço de transporte público da cidade do Recife, Brasil. A análise é feita por meio de técnicas de estatística descritiva a fim de conhecer o comportamento dos ônibus e qual o impacto as chuvas podem causar na dinâmica das velocidades.

A cidade do Recife apresenta um perfil interessante para este estudo pois é um local propício a ocorrência de alagamentos por diversas causas: chuvas torrenciais, inundações fluviais, planície de baixa altitude e marés altas de maior amplitude [Cabral and Alencar 2005]. Além disso, a estrutura viária apresenta problemas conhecidos em pontos de alagamento.

A principal contribuição deste trabalho é, portanto, responder as seguintes perguntas no contexto da cidade do Recife: Como os níveis de chuva afetam a dinâmica do serviço de ônibus? Se afetam, como medir o impacto quantitativamente principalmente com relação à velocidade?

Este trabalho está organizado da seguinte forma: (i) a seção 2 apresenta uma revisão da literatura de trabalhos que trazem abordagens de análise de tráfego com mudanças climáticas; (ii) seção 3 descreve qual o conjunto de dados utilizado; (iii) a seção 4 descreve a metodologia utilizada para analisar os dados; (iv) na seção 5 os resultados da análise são discutidos e (iv) na seção 6 conclusões e trabalhos futuros.

\section{Trabalhos relacionados}

Nesta seção são apresentados alguns trabalhos que analisam o impacto de mudanças climáticas no trânsito. Os impactos podem causar variações no fluxo de veículos, velocidade, número de acidentes, densidade (número de veículos em um determinado comprimento da rodovia), tempo médio de viagem, etc.

[Hofmann and O'Mahony 2005] realiza um trabalho com objetivo de investigar o impacto das condições climáticas no transporte urbano de forma que se possa medir o comportamento dos passageiros que utilizam o serviço. Para alcançar tal objetivo, o trabalho propõe utilizar dados de meteorologia e de ônibus de forma a traçar correlações de forma que se possa medir alguns fatores: quantidade de pessoas que utilizam o serviço público (ridership), frequência do serviço (disponibilidade do serviço), a distância entre os ônibus em uma rota (headways), agrupamento de ônibus (bus bunching), tempo de viagem e a variabilidade do tempo de viagem. Todos os fatores são analisado levando em consideração dois cenários: dias com chuva e sem chuva. De acordo com o autor, a chuva

\footnotetext{
${ }^{2}$ http://www.bikely.com/

${ }^{3}$ http://research.microsoft.com/en-us/projects/geolife/

${ }^{4}$ www.facebook.com
} 
influencia negativamente as medidas ridership e travel time, entretanto nas medidas de bus bunching e headway, a chuva contribui positivamente, ou seja, menos agrupamento e melhora na frequência dos ônibus.

[Cools et al. 2010] objetiva examinar se as condições climáticas alteram uniformemente a intensidade do tráfego na Bélgica. De acordo com os autores, alguns fatores do tempo como chuva, neve, neblina e outros, influenciam vários aspectos do tráfego, por exemplo, intensidade do tráfego, velocidade etc. Para avaliar os efeitos do clima, os autores utilizaram dados reais providos por inductive loop detector que coletou dados durante o período de 1 ano (2003-2004). Para avaliar o impacto que as variações climáticas causam no trânsito, o trabalho utilizou a técnica de correlação não paramétrica de Spearman e a técnica de Regressão Linear. Os resultados mostraram que chuvas, nebulosidade e ventania são negativamente correlacionadas com a intensidade do tráfego, enquanto boas condições climáticas (dias ensolarados) são proporcionais a intensidade do tráfego. $\mathrm{O}$ trabalho apresentou os seguintes resultados: a intensidade do tráfego é maior quando ocorre máximas temperaturas; o impacto das condições climáticas são claramente mais homogêneas em locais próximos de onde as informações foram coletadas do que em diferentes locais. Também foi notado que rodovias que são mais usadas para lazer são mais suscetíveis às mudanças de tráfego devido às variações climáticas do que àquelas avenidas que são utilizadas para acesso casa -> trabalho, trabalho -> casa.

[Chung et al. 2005] apresentam uma análise do impacto das chuvas na cidade de Tóquio (Japão). Os autores utilizam dados reais tanto de meteorologia quanto de tráfego. Os dados foram coletados durante 6 anos e a análise foi feita apenas considerando a via arterial (Tokyo Metropolitan Expressway). Para realizar a análise, o trabalho configurou o experimento da seguinte forma: dias da semana (segunda até sábado) e feriados (domingos e feriados de fato). Os autores realizaram a análise levando em consideração dias com chuva e sem chuva. Um dia só foi considerado chuvoso se a precipitação fosse maior ou igual a $13 \mathrm{~mm}$, assim, 216 dias foram considerados chuvoso num espaço de 6 anos. Os resultados mostraram que os dias de sábado e domingo são mais sensíveis no sentido de haver um decrescimento na quantidade de viagem quando ocorre chuva. O trabalho também encontrou uma correlação positiva do número de acidentes nos finais de semana e que a frequência de acidentes em dias chuvosos é maior do que nos outros dias.

[Andersson and Chapman 2011] propõem analisar acidentes de tráfego no município West Midlands, Reino Unido, durante o inverno com o objetivo de aplicar cenários de mudança climática da UKCIP (UK Climate Impact Program) para estimar mudanças quanto ao número de acidentes no tráfego, juntamente com dados de clima gerados artificialmente com base nos cenários. Os dados foram gerados levando em consideração dois modelos estocásticos usados pelo EARWIG (Environment Agency Rainfall and Weather Impacts Generator). Os resultados dos cenários (2020, 2050 e 2080 anos) mostram que a quantidade de dias com temperaturas abaixo de $0^{\circ}$ e $5^{\circ}$ tendem a diminuir, proporcionando dias mais quentes. Foi encontrada uma correlação entre a redução de dias com temperatura abaixo de $5^{\circ} \mathrm{e}$ a redução do número de acidentes. Mesmo notando tal correlação, outras variáveis que não são levadas em consideração podem afetar os resultados. $\mathrm{O}$ trabalho conclui que o número de dias com temperatura abaixo de $0^{\circ}$ diminuirão no futuro e isto reduzirá de $43 \%$ no número de acidentes em West Midlands.

Embora os trabalhos citados apresentem análise sobre como as condições climáticas podem influenciar o comportamento dos usuários de transportes urbanos [Hofmann and O'Mahony 2005], a intensidade do tráfego [Cools et al. 2010], a quantidade de acidentes [Chung et al. 2005], [Andersson and Chapman 2011], nenhum deles, 
até o nosso atual conhecimento, propõem-se a verificar pontualmente o quanto a velocidade média dos transportes públicos (ônibus) aumentam ou diminuem de acordo com os níveis de precipitação pluviométrica. Informações pontuais, como as produzidas neste trabalho podem ser utilizadas de forma precisa pelos gestores de tráfego viário para realizar tomadas de decisão.

\section{Conjunto de Dados}

Os dados do sistema de ônibus foram fornecidos pelo Grande Recife Consórcio de Transporte Metropolitano ${ }^{5}$ que gerencia o transporte de ônibus na região metropolitana do Recife, e incluem coordenadas das rotas, registros de GPS dos ônibus, os quais denominamos de ping. Cada ping contém os identificadores da linha e rota, localização e velocidade dos ônibus. O conjunto de dados disponibilizado abrange apenas o período entre 17/10/2017 e 24/01/2018, possui 129 linhas e 891 ônibus. Os ônibus emitem um ping a uma frequência de 30 segundos, e a base disponibilizada para este trabalho tem 73 milhões de pings. Estes pings estão visualmente representados em vermelho no mapa da Figura 1, o qual apresenta em destaque o perímetro do município do Recife.

Os dados pluviométricos foram obtidos no site do CEMADEN (Centro Nacional de Monitoramento e Alertas de Desastres Naturais ${ }^{6}$ ), o qual disponibiliza o volume de chuvas acumuladas a cada hora em cada uma das 18 estações pluviométricas que estão geograficamente distribuídas pela cidade. As localizações das estações pluviométricas também são fornecidas e estão apresentadas com a cor alaranjada no mapa da Figura 1.

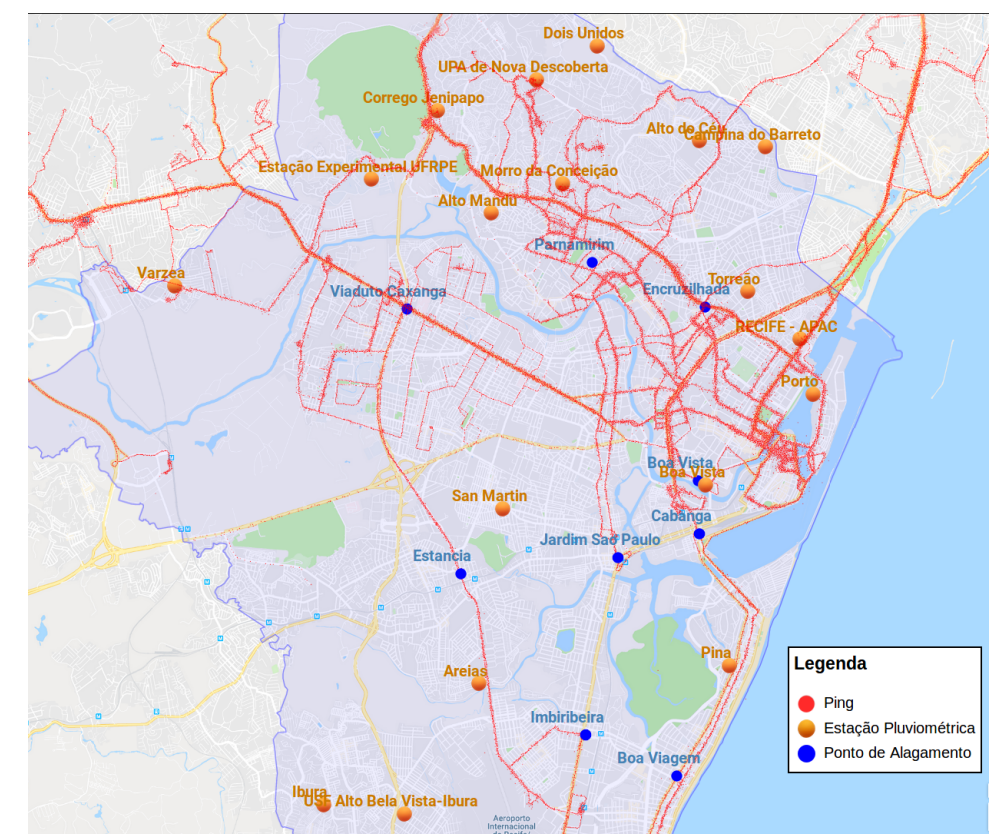

Figura 1. Localização do Conjunto de Dados Georreferenciados

Este trabalho envolve a análise do impacto das chuvas no transporte público em regiões críticas de alagamento do Recife, as regiões críticas de alagamento foram obtidas em um estudo da Emlurb (Empresa de Manutenção e Limpeza Urbana) [EMLURB 2013] que catalogou 159 pontos de alagamentos. Todos os dados sobre ônibus, pluviômetros e

\footnotetext{
${ }^{5}$ http://www.granderecife.pe.gov.br/web/grande-recife

${ }^{6} \mathrm{http}: / /$ www.cemaden.gov.br/pluviometrosautomaticos/
} 
locais críticos de alagamento foram inseridos em um banco de dados relacional fazendo uso dos recursos geográficos e índices espaciais, sob o qual as análises foram realizadas.

\section{Método}

Nesta seção apresentamos a abordagem utilizada para avaliar o impacto das chuvas na velocidade média dos ônibus. Para isso, foram realizadas duas tarefas: limpeza e seleção dos dados, e a análise em três etapas.

\subsection{Limpeza e Seleção dos Dados}

A limpeza dos dados é importante para removermos valores com problemas, tais como dados nulos e valores atípicos (outliers) que podem afetar a análise. Para esta tarefa realizamos um processo semi-automatizado em que removemos os registros com problema. Os dados dos ônibus foram disponibilizados em formato CSV (Comma Separated Values), e do total de 73 milhões de pings, somente 26 milhões puderam ser aproveitados para os objetivos deste trabalho, pois os demais apresentavam dados de velocidade, localização ou linha, nulos. Os dados dos pluviômetros da CEMADEN não apresentaram problemas relevantes e puderam ser importados automaticamente de um arquivo CSV para a base relacional. Os dados dos pontos de alagamento foram disponibilizados em formato de relatório pela Emlurb, e foi necessário um processo manual de importação.

Após a limpeza, foi realizada a seleção dos dados com propósito de delimitar a análise em dias úteis, e assim os feriados e fins de semana foram removidos. Pois, em principio, estes apresentam comportamento atípico, em que o trânsito é menos intenso e a velocidade média dos ônibus é bastante superior em comparação aos dias úteis. Dentro desse subconjunto foram selecionados os dados de pluviômetros e velocidades de ônibus apenas na faixa de horário entre $6 \mathrm{~h}$ e $20 \mathrm{~h}$, pois entre $20 \mathrm{~h}$ e $6 \mathrm{~h}$ a quantidade de ônibus circulando é menor.

Por fim, dos 159 locais de alagamento apresentados pela Emlurb foram selecionados os 8 locais considerados mais críticos. Os critérios para seleção são os locais em que circulam mais ônibus e que estão próximos a vias arteriais principais e secundárias, e cruzamentos de avenidas, sendo representados com a cor azul no mapa da Figura 1.

\subsection{Processo de Análise}

A ideia de análise proposta é verificar o impacto do volume de chuvas na velocidade média dos ônibus perto dos locais de alagamento, esta região é delimitada por uma circunferência de raio $R$. Dentro da região comparamos a velocidade média registrada para cada linha de ônibus em horários de chuva versus a média dos dados históricos.

Na primeira etapa da análise, para cada local de alagamento foi calculada a velocidade média histórica e o desvio padrão do histórico dos pings enviados dentro da região nos dias em que não ocorreu chuva, este cálculo discrimina dia da semana, faixa de hora e linha de ônibus. Na segunda etapa, uma data alvo e um local alvo são escolhidos para análise, neste trabalho as datas alvos são dias de ocorrência de chuvas e os locais são regiões críticas de alagamento. Mais especificamente, é calculada a velocidade média registrada pelos ônibus na região do local alvo na data alvo. Este cálculo é realizado a cada janela de uma hora e discrimina os valores para cada linha de ônibus. Na terceira etapa, selecionamos estações pluviométricas próximas ao local alvo de análise e realizamos as médias dos acumulados de chuvas agrupando os dados em faixas de uma hora.

A Tabela 1 apresenta um exemplo dos dados resultantes do processo de análise da Linha 644 - Largo Maracanã perto do local Encruzilhada no dia 19/01/2018. Nota-se que 


\begin{tabular}{|c|c|c|c|c|c|c|c|c|c|c|c|c|c|c|c|}
\hline \multirow[b]{2}{*}{ Variáveis } & \multicolumn{15}{|c|}{ HORA } \\
\hline & 6 & 7 & 8 & 9 & 10 & 11 & 12 & 13 & 14 & 15 & 16 & 17 & 18 & 19 & 20 \\
\hline $\operatorname{Max}(\mathrm{Km} / \mathrm{h})$ & 19 & 17 & 14 & 13 & 15 & 15 & 15 & 16 & 17 & 15 & 18 & 17 & 17 & 20 & 21 \\
\hline $\operatorname{Min}(\mathrm{Km} / \mathrm{h})$ & 14 & 8 & 7 & 8 & 10 & 10 & 9 & 9 & 9 & 11 & 8 & 7 & 9 & 13 & 16 \\
\hline Mediana & 15 & 9 & 10 & 11 & 12,5 & 11,5 & 10,5 & 12,5 & 13,5 & 12,5 & 13 & 12 & 12,5 & 16 & 18,5 \\
\hline Média $(\mathrm{Km} / \mathrm{h})$ & 15,7 & 11,4 & 10,1 & 10,9 & 12,3 & 12 & 11,4 & 12,5 & 13,1 & 12,8 & 13,3 & 11,9 & 13,3 & 16,5 & 18,3 \\
\hline Desvio Padrão & 1,7 & 3,3 & 2,1 & 1,4 & 1,9 & 1,9 & 2,1 & 2,5 & 2,8 & 1,2 & 2,8 & 2,8 & 3 & 1,9 & 1,8 \\
\hline Vel. Registrada 19/01/18 & 15 & 15 & 5 & 6 & 9 & 14 & 16 & 16 & 16 & 17 & 14 & 16 & 15 & 20 & 19 \\
\hline Acumulado Chuvas (mm) & 4 & 5,2 & 10,5 & 12,4 & 8,2 & 1,5 & 0 & 0 & 0 & 0 & 0 & 0 & 0,2 & 0 & 0 \\
\hline
\end{tabular}

Tabela 1: 19/1/2018 - Encruzilhada - Linha 644

nesta data e local a linha 644 registrou uma velocidade de $5 \mathrm{Km} / \mathrm{h}$ às $8 \mathrm{~h}$ enquanto que a média histórica das sextas-feiras nesse mesmo horário é $10.1 \mathrm{Km} / \mathrm{h}$.

Para a realização efetiva das análises foi desenvolvido um sistema em linguagem Python ${ }^{7}$ fazendo uso da biblioteca Pandas ${ }^{8}$ que disponibiliza um conjunto de estruturas de dados e funções estatísticas apropriados para softwares do contexto de ciência de dados. Por fim, a Figura 2 sintetiza a metodologia descrita nesta seção.

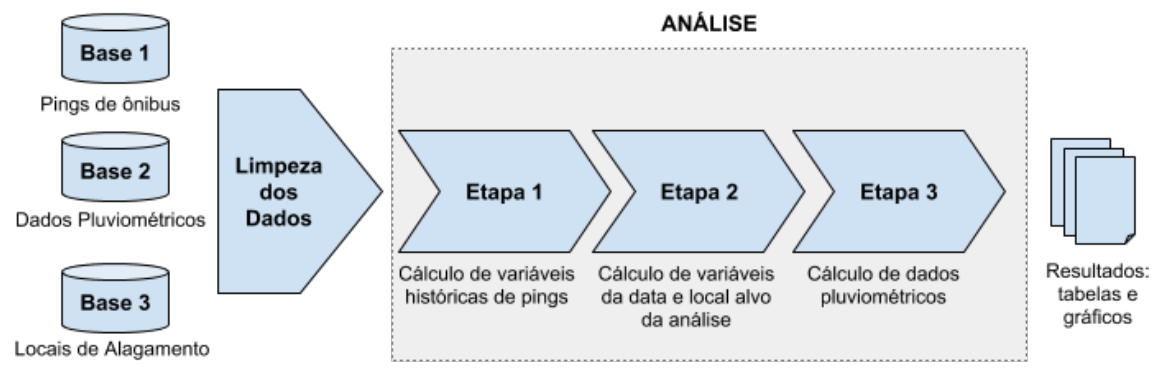

Figura 2. Abordagem utilizada para seleção e análise dos dados.

\section{Análises}

A abordagem de análise foi aplicada e para tal foi selecionada a data 19/01/2018, pois nesta data ocorreram chuvas com volumes significativos, com acumulados maiores que 10 milímetros por hora em diversas estações pluviométricas. Dentro do período de abrangência dos dados disponibilizados não há outras ocorrências de volume de chuvas de maior intensidade. Foi selecionado o local Viaduto da Caxangá para detalhar a análise, pois é um local próximo às estações pluviométricas que registraram maior volume de chuvas e também é um ponto conhecido por problemas de trânsito em dias de chuva.

\subsection{Configurações da Análise}

O processo e análise possui algumas variáveis que podem ser configuradas, tais como, faixa de horário diária, remoção de fins de semana, remoção de feriados e o raio da região de análise.

As análises são realizadas nas regiões dos locais típicos de alagamento, e estas regiões são delimitadas por uma circunferência de raio $R$. Foram realizados alguns testes empíricos com diferentes valores de raio $R$ com intervalos de 100 metros. Os testes demonstraram que $R<400$ não incluem dados suficientes de pings de ônibus que circulam na região, deixando a análise com muitos dados faltantes em algumas faixas de horário. Para $R \geq 1000$ metros são incluídos muitos dados de ônibus que não são afetados pelo

\footnotetext{
${ }^{7}$ https://www.python.org/

${ }^{8}$ https://pandas.pydata.org/
} 
alagamento, assim os desvios de velocidade média são suavizados por pings que não sofrem impacto por estarem muito longe do local alvo. Foi adotado um raio de 700 metros, pois apresenta um equilíbrio entre os parâmetros com uma quantidade pings satisfatória.

Por fim, para cada local de alagamento foram selecionadas estações pluviométricas próximas. A disposição geográfica das estações na cidade é bastante irregular, como ilustrado na Figura 1, havendo concentração de estações em algumas regiões e poucas estações em outras. Por esta razão foi adotada uma distância bastante abrangente, assim as estações pluviométricas que se localizam a um $R<4500$ metros do local de alagamento são inclusas na análise.

\subsection{Análise do Dia 19/01/2018}

O volume de chuvas da sexta-feira dia 19/01/2018 pode ser observado na Tabela 2 . Todas as estações pluviométricas registraram chuvas maiores que $10 \mathrm{~mm}$, sendo que metade das estações chegou a $20 \mathrm{~mm}$, e em específico a estação Várzea atingiu $36 \mathrm{~mm}$. As chuvas se concentraram na faixa de horário de $6 \mathrm{~h}$ às $11 \mathrm{~h}$.

\begin{tabular}{lcccccccccccccccc}
\hline & & \multicolumn{11}{c}{ HORA } \\
\hline Estação & $\mathbf{6}$ & $\mathbf{7}$ & $\mathbf{8}$ & $\mathbf{9}$ & $\mathbf{1 0}$ & $\mathbf{1 1}$ & $\mathbf{1 2}$ & $\mathbf{1 3}$ & $\mathbf{1 4}$ & $\mathbf{1 5}$ & $\mathbf{1 6}$ & $\mathbf{1 7}$ & $\mathbf{1 8}$ & $\mathbf{1 9}$ & $\mathbf{2 0}$ & Acumulado \\
Várzea & 6 & 1 & 16 & 36 & 12 & 2 & 0 & 0 & 0 & 0 & 0 & 0 & 0 & 0 & 0 & 73 \\
Córrego Jenipapo & 6 & 2 & 13 & 16 & 17 & 2 & 0 & 0 & 0 & 0 & 0 & 0 & 3 & 1 & 0 & 60 \\
Alto Mandu & 5 & 4 & 18 & 19 & 10 & 2 & 0 & 0 & 0 & 0 & 0 & 0 & 1 & 0 & 0 & 59 \\
UPA de Nova Descoberta & 7 & 3 & 10 & 21 & 15 & 2 & 0 & 0 & 0 & 0 & 0 & 0 & 1 & 0 & 0 & 59 \\
Areias & 2 & 0 & 21 & 27 & 3 & 3 & 0 & 0 & 0 & 0 & 0 & 0 & 0 & 0 & 0 & 56 \\
Estação Experimental UFRPE & 4 & 2 & 17 & 15 & 13 & 2 & 0 & 0 & 0 & 0 & 0 & 0 & 0 & 0 & 0 & 53 \\
Alto do Céu & 5 & 8 & 6 & 13 & 12 & 1 & 0 & 0 & 0 & 0 & 0 & 0 & 0 & 0 & 0 & 45 \\
San Martin & 2 & 2 & 15 & 24 & 4 & 3 & 0 & 0 & 0 & 0 & 0 & 0 & 0 & 0 & 0 & 50 \\
Morro da Conceição & 5 & 4 & 13 & 14 & 12 & 1 & 0 & 0 & 0 & 0 & 0 & 0 & 1 & 0 & 0 & 50 \\
USF Alto Bela Vista-Ibura & 0 & 0 & 13 & 24 & 2 & 3 & 0 & 0 & 0 & 0 & 0 & 0 & 0 & 0 & 0 & 42 \\
Campina do Barreto & 6 & 11 & 4 & 11 & 9 & 1 & 0 & 0 & 0 & 0 & 0 & 0 & 0 & 0 & 0 & 42 \\
Porto & 5 & 8 & 7 & 11 & 8 & 1 & 0 & 0 & 0 & 0 & 0 & 0 & 0 & 0 & 0 & 40 \\
Torreão & 5 & 6 & 8 & 10 & 10 & 1 & 0 & 0 & 0 & 0 & 0 & 0 & 0 & 0 & 0 & 40 \\
Pina & 0 & 4 & 19 & 11 & 2 & 2 & 0 & 0 & 0 & 0 & 0 & 0 & 0 & 0 & 0 & 38 \\
Dois Unidos & 5 & 5 & 10 & 10 & 7 & 0 & 0 & 0 & 0 & 0 & 0 & 0 & 0 & 0 & 0 & 37 \\
Ibura & 0 & 0 & 13 & 20 & 1 & 3 & 0 & 0 & 0 & 0 & 0 & 0 & 0 & 0 & 0 & 37 \\
Boa Vista & 3 & 2 & 11 & 8 & 4 & 2 & 0 & 0 & 0 & 0 & 0 & 0 & 0 & 0 & 0 & 30 \\
Recife - APAC & 1 & 5 & 8 & 2 & 9 & 1 & 0 & 0 & 0 & 0 & 0 & 0 & 0 & 0 & 0 \\
\hline
\end{tabular}

Tabela 2: Chuvas em milímetros - 19/1/2018 - Acumulado em frequência 1 hora

A Tabela 3 apresenta os dados do dia 19/01/2018 com os locais de alagamento que demonstram diferenças mais expressivas na velocidade média, tais como, Viaduto Caxangá, Encruzilhada, Parnamirim e Boa Vista, enquanto os demais locais apresentaram impactos menos contundentes e não foram inclusos na tabela.

\begin{tabular}{|c|c|c|c|c|c|c|c|c|c|c|c|c|c|c|c|c|}
\hline \multirow[b]{2}{*}{ Local } & \multirow[b]{2}{*}{ Variáveis } & \multicolumn{15}{|c|}{ HORA } \\
\hline & & 6 & 7 & 8 & 9 & 10 & 11 & 12 & 13 & 14 & 15 & 16 & 17 & 18 & 19 & 20 \\
\hline \multirow{6}{*}{ Viaduto Caxangá } & $\operatorname{Max}$ & 20,7 & 19,7 & 19,3 & 20,7 & 20 & 20,3 & 20 & 22 & 21 & 19,7 & 24,7 & 19 & 18 & 21,3 & 22,3 \\
\hline & Min & 17,7 & 16,3 & 18,3 & 18,3 & 17,3 & 17,7 & 15,5 & 16 & 15,7 & 15,7 & 16 & 14 & 11,7 & 15 & 13,7 \\
\hline & Mediana & 19,5 & 18,7 & 18,8 & 19,2 & 18,8 & 19 & 18 & 18,3 & 18,2 & 17,5 & 16,7 & 16,5 & 15,2 & 17,7 & 20,7 \\
\hline & Média & 19,3 & 18,3 & 18,8 & 19,3 & 18,7 & 19,1 & 17,9 & 18,6 & 18,4 & 17,4 & 17,9 & 16,2 & 15,3 & 18,1 & 19,8 \\
\hline & Desvio Padrão & 1,1 & 1,3 & 0,3 & 0,9 & 0,9 & 1,3 & 1,7 & 2,6 & 2,1 & 1,6 & 3,4 & 1,8 & 2,2 & 2,5 & 3,2 \\
\hline & V. R. 19/1/2018 & 18 & 19 & 16,7 & 16 & 9,3 & 7,7 & 16,7 & 19 & 17,3 & 15,3 & 16 & 17 & 17,7 & 19,7 & 22 \\
\hline \multirow{6}{*}{ Boa Vista } & Max & 26 & 24 & 26 & 23 & 26 & 25 & 27 & 28 & 23 & 22 & 23 & 20 & 22 & 24 & 26 \\
\hline & Min & 22 & 15 & 17 & 18 & 16 & 19 & 18 & 19 & 14 & 11 & 11 & 10 & 8 & 13 & 18 \\
\hline & Mediana & 24 & 20 & 22 & 21 & 22,5 & 20 & 23,5 & 24 & 16,5 & 16,5 & 13,5 & 11,5 & 12 & 18 & 23 \\
\hline & Média & 23,7 & 20,1 & 21,1 & 20,7 & 21,8 & 21,4 & 22,9 & 23,6 & 18,2 & 16,7 & 15,4 & 13,2 & 14 & 17,9 & 23 \\
\hline & Desvio Padrão & 1,3 & 2,8 & 2,7 & 1,8 & 3 & 2,2 & 2,7 & 2,8 & 3,2 & 3,2 & 3,9 & 3,5 & 4,9 & 3,1 & 2,4 \\
\hline & V. R. $19 / 1 / 2018$ & 20 & 21 & 8 & 16 & 22 & 22 & 21 & 24 & 21 & 21 & 21 & 19 & 19 & 21 & 28 \\
\hline \multirow{6}{*}{ Encruzilhada } & $\operatorname{Max}$ & 19,7 & 17,3 & 15,7 & 14,7 & 16 & 17,7 & 17,7 & 18,7 & 17,7 & 17,7 & 17,3 & 16,3 & 18,3 & 21 & 23 \\
\hline & Min & 15 & 9 & 8,3 & 10,3 & 7,7 & 11,7 & 11,7 & 10,3 & 9,7 & 12 & 10,3 & 10,3 & 12 & 13,3 & 18,3 \\
\hline & Mediana & 16,3 & 10 & 10 & 12,5 & 14 & 14,7 & 12,8 & 15 & 14,2 & 15,8 & 14,7 & 12,8 & 13,2 & 17,3 & 21,2 \\
\hline & Média & 16,7 & 12,2 & 11,3 & 12,6 & 13,4 & 14,6 & 13,9 & 14,7 & 14,1 & 15,4 & 14,5 & 13,2 & 14,5 & 17,5 & 20,9 \\
\hline & Desvio Padrão & 1,5 & 3,4 & 2,5 & 1,5 & 2,6 & 2 & 2,3 & 2,8 & 2,8 & 1,8 & 2,2 & 2,1 & 2,4 & 2,2 & 1,4 \\
\hline & V. R. $19 / 1 / 2018$ & 17 & 14 & 7,3 & 7,7 & 10,7 & 16 & 17,7 & 16,7 & 19,3 & 17 & 15,7 & 16,7 & 15,3 & 20,3 & 21,3 \\
\hline
\end{tabular}

Tabela 3: Velocidade Média Registrada nos Locais de Alagamento no dia 19/1/2018

Um caso interessante é o local Viaduto da Caxangá às $11 \mathrm{~h}$, quando a velocidade registrada no dia 19/01/2018 foi $7,7 \mathrm{Km} / \mathrm{h}$ bastante inferior aos $17,7 \mathrm{Km} / \mathrm{h}$ que é a mínima 
histórica dos dias que não choveu. O gráfico da Figura 3 apresenta a média em milímetros da intensidade de chuva das estações pluviométricas impactantes, e também o comportamento histórico da velocidade média, desvio padrão em dias que não ocorreu chuva e a velocidade registrada no dia 19/01/2018. No gráfico nota-se que, na faixa de horário de $8 \mathrm{~h}$ as $11 \mathrm{~h}$, o comportamento da velocidade média no dia 19/01/2018 se manteve inferior à média histórica: $34 \%$ menor que a média histórica para esta faixa de horário.

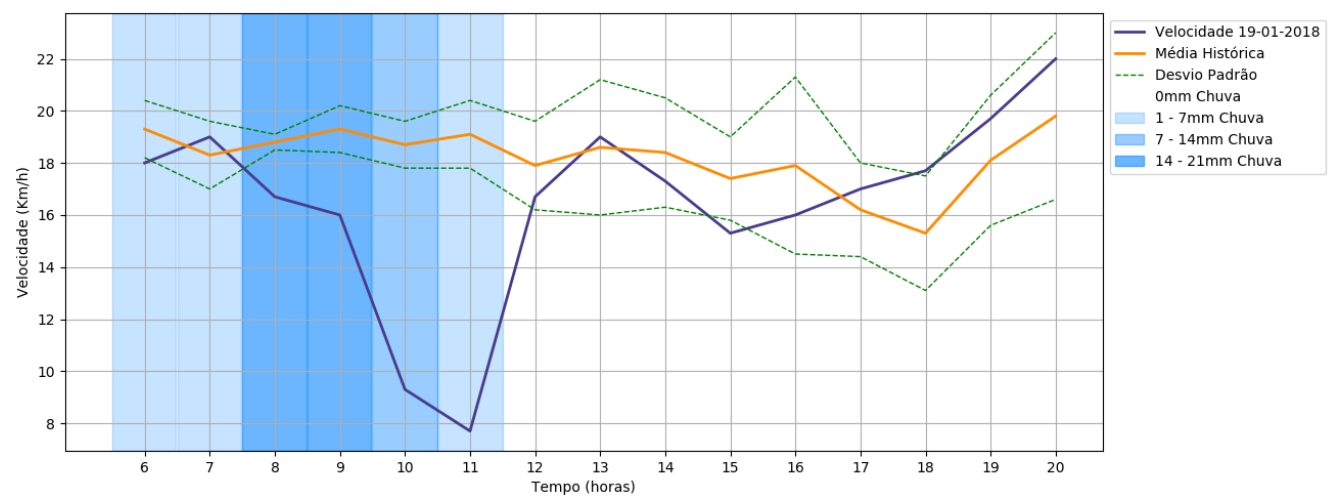

Figura 3. Velocidade Média no Viaduto da Caxangá no dia 19/1/2018

A linha 2480 TI Camaragipe/Derby BRT foi selecionada para uma análise isolada. A Figura 4 exibe em amarelo a circunferência com raio de 700 metros do ponto de alagamento e em verde o percurso de linha 2480 na região do local Viaduto Caxangá. Observa-se que o trajeto da linha passa pela avenida Caxangá e cruzando a BR101 por baixo do viaduto nas rotas de ida e volta. A distância percorrida é de 1400 metros na ida e 1400 metros na volta. A tabela 4 apresenta os dados históricos de máximo, mínimo, média, mediana, desvio padrão, e os dados de acumulados de chuvas e velocidade registrada no dia 19/01/2018. A Figura 5 apresenta o gráfico com os dados de chuvas, velocidade média registrada no dia 19/01/2018 e velocidades médias

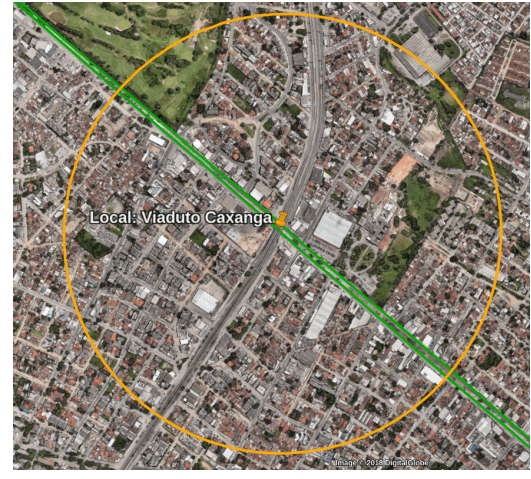

Figura 4. Trajeto Linha 2880 - Viaduto Caxangá e desvios padrões históricos para o local Viaduto Caxangá.

\begin{tabular}{|c|c|c|c|c|c|c|c|c|c|c|c|c|c|c|c|}
\hline \multirow[b]{2}{*}{ Variáveis } & \multicolumn{15}{|c|}{ HORA } \\
\hline & 6 & 7 & 8 & 9 & 10 & 11 & 12 & 13 & 14 & 15 & 16 & 17 & 18 & 19 & 20 \\
\hline $\operatorname{Max}(\mathrm{Km} / \mathrm{h})$ & 23 & 22 & 22 & 23 & 22 & 25 & 22 & 32 & 23 & 22 & 21 & 22 & 22 & 21 & 22 \\
\hline $\operatorname{Min}(\mathrm{Km} / \mathrm{h})$ & 18 & 18 & 20 & 20 & 18 & 18 & 17 & 12 & 16 & 18 & 17 & 17 & 13 & 18 & 16 \\
\hline Mediana & 21,5 & 19,5 & 21 & 21,5 & 21 & 21 & 20 & 22 & 20 & 20,5 & 19,5 & 18,5 & 19,5 & 20 & 21,5 \\
\hline Média $(\mathrm{Km} / \mathrm{h})$ & 20,8 & 19,8 & 21 & 21,5 & 20,5 & 21,2 & 19,8 & 21,6 & 19,6 & 20,2 & 19,3 & 19 & 18,2 & 19,7 & 20,5 \\
\hline Desvio Padrão & 1,9 & 1,5 & 0,9 & 1 & 1,4 & 2,6 & 1,9 & 7,4 & 2,5 & 1,5 & 1,4 & 2,1 & 3,8 & 1,4 & 2,3 \\
\hline Vel. Registrada 19/01/18 & 20 & 20 & 21 & 19 & 13 & 5 & 22 & 22 & 24 & 20 & 20 & 22 & 22 & 23 & 24 \\
\hline Acumulado Chuvas (mm) & 4,7 & 2,5 & 15,3 & 20,7 & 11,3 & 2 & 0 & 0 & 0 & 0 & 0 & 0 & 0,8 & 0,2 & 0 \\
\hline
\end{tabular}

Tabela 4: Dados Linha 2480 Viaduto Caxangá dia 19/1/2018

Ao analisar a linha 2480 em isolado, observa-se que, durante o período de $9 \mathrm{~h}$ às $11 \mathrm{~h}$, a velocidade média diminuiu além do desvio padrão. Ao comparar a velocidade média registrada em 19/01/2018 de 14,5Km/h há uma diminuição de $31 \%$ em relação à média histórica que é $21,05 \mathrm{Km} / \mathrm{h}$, isso reflete no tempo médio para realizar o percurso 


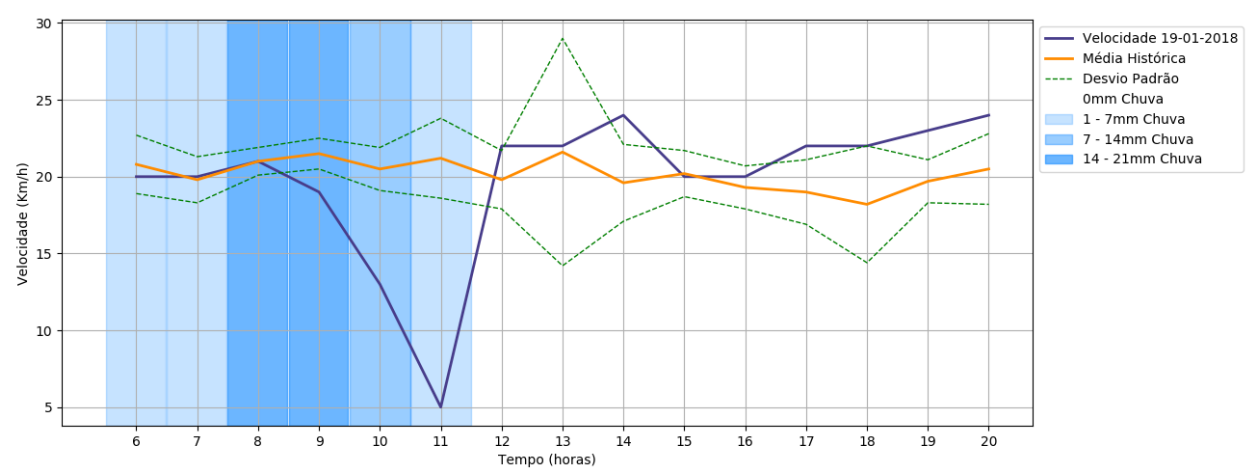

Figura 5. Velocidade Média Linha 2480 no Viaduto da Caxangá em 19/1/2018

de 1400 metros que tipicamente em uma sexta-feira sem chuva é de 4 minutos e em 19/01/2018 foi de 5 minutos 42 segundos.

Uma ressalva é que os resultados foram obtidos com base em períodos que não refletem o cotidiano da cidade, os meses de Dezembro e Janeiro são bastante atípicos e há diversos fatores que influenciam na variança dos dados, tais como, férias escolares, feriados etc. Outro ponto importante é o regime de chuvas do verão, há poucos eventos de chuva relevantes para o trabalho e assim não foi possível realizar análises mais variadas e avançadas. Mesmo com estes pontos a serem considerados, as análises realizadas trouxeram resultados que estão alinhados com a ideia de que chuvas provocam problemas de trânsito nos locais estudados. No caso específico do dia 19/01/2018, há notícias que evidenciam a ocorrência de problemas de tráfego que prejudicam o transporte público. [G1 2018]

\section{Conclusão e Trabalhos Futuros}

Este trabalho apresentou um processo de análise de dados do sistema de ônibus baseado em estatística descritiva, e focou a identificação de impactos que as chuvas podem causar na velocidade média dos ônibus. Como contribuição principal a metodologia utilizada se mostrou efetiva em responder as questões inciais sobre como os níveis de chuva afetam a dinâmica do serviço de ônibus e como medir o impacto quantitativamente com relação à velocidade. Foi possível identificar a variação da velocidade média dos ônibus em um dia de chuva. A análise da linha 2480 na região Viaduto da Caxangá no dia 19/01/2018 apresentou uma redução de $31 \%$ da velocidade média em relação aos dados históricos de dias sem chuva.

Como trabalhos futuros o processo de análise desenvolvido pode ser replicado em outras cidades com objetivo de comparação. A análise também pode ser expandida para verificar os impacto de outras variáveis, como acidentes de trânsito, fluxo de veículos, horários de pico, grande eventos, obras públicas, entre outros. Atualmente está em andamento a disponibilização do sistema de software em interface Web que possa ser utilizado por gestores para análise e suporte à tomada de decisão no contexto de transporte público. Por fim, os trabalhos futuros devem envolver a análise de mais variáveis e o uso de outras técnicas estatísticas, tais como a realização de testes de hipóteses e correlações entre locais de redução de velocidade média e o volume de chuvas em estações pluviométricas.

\section{Agradecimentos}

Esta pesquisa foi parcialmente financiada pelo INES 2.0, FACEPE PRONEX APQ 03881.03/14 e CNPq 465614/2014-0. Os autores agradecem a Fernando Guedes e Alexandre 
Severo, do Grande Recife Consórcio de Transporte Metropolitano, pelo provimento dos dados utilizados nesta análise.

\section{Referências}

Andersson, A. K. and Chapman, L. (2011). The impact of climate change on winter road maintenance and traffic accidents in west midlands, uk. Accident Analysis \& Prevention, 43(1):284-289.

Cabral, J. J. S. P. and Alencar, A. V. (2005). Recife e a convivência com as águas. In Gestão do Território e Manejo Integrado das águas urbanas, pages 109-117.

Chung, E., Ohtani, O., Warita, H., Kuwahara, M., and Morita, H. (2005). Effect of rain on travel demand and traffic accidents. In Intelligent Transportation Systems, 2005. Proceedings. 2005 IEEE, pages 1080-1083. IEEE.

Cools, M., Moons, E., and Wets, G. (2010). Assessing the impact of weather on traffic intensity. Weather, Climate, and Society, 2(1):60-68.

EMLURB (2013). Elaboração dos estudos de concepção para gestão e manejo de águas pluviais e drenagem urbana do recife (versão concedida em visita técnica). Empresa de Manutenção e Limpeza Urbana.

G1 (2018). Chuva alaga ruas e avenidas do grande recife. disponível em: $<$ https://g1.globo.com/pe/pernambuco/noticia/chuvas-causam-alagamentos-etranstornos-no-transito-no-grande-recife.ghtml> acesso 2 de abril de 2018.

Hofmann, M. and O'Mahony, M. (2005). The impact of adverse weather conditions on urban bus performance measures. In Intelligent Transportation Systems, 2005. Proceedings. 2005 IEEE, pages 84-89. IEEE.

Keay, K. and Simmonds, I. (2006). Road accidents and rainfall in a large australian city. Accident Analysis \& Prevention, 38(3):445-454.

Koetse, M. J. and Rietveld, P. (2009). The impact of climate change and weather on transport: An overview of empirical findings. Transportation Research Part D: Transport and Environment, 14(3):205-221.

Pan, B., Zheng, Y., Wilkie, D., and Shahabi, C. (2013). Crowd sensing of traffic anomalies based on human mobility and social media. Proceedings of the 21 st ACM SIGSPATIAL International Conference on Advances in Geographic Information Systems - SIGSPATIAL'13, pages 334-343.

Pelekis, N., Kopanakis, I., Marketos, G., Ntoutsi, I., Andrienko, G., and Theodoridis, Y. (2007). Similarity Search in Trajectory Databases. 14th International Symposium on Temporal Representation and Reasoning (TIME'07), pages 129-140.

Savage, N. S., Nishimura, S., Chavez, N. E., and Yan, X. (2010). Frequent trajectory mining on GPS data. Proceedings of the 3rd International Workshop on Location and the Web - LocWeb '10, pages 1-4.

Smith, B. L., Byrne, K. G., Copperman, R. B., Hennessy, S. M., and Goodall, N. J. (2004). An investigation into the impact of rainfall on freeway traffic flow. In 83rd annual meeting of the Transportation Research Board, Washington DC. Citeseer.

Songchitruksa, P. and Balke, K. (2006). Assessing weather, environment, and loop data for real-time freeway incident prediction. Transportation Research Record: Journal of the Transportation Research Board, (1959):105-113. 